\title{
Tocilizumab in the Treatment of Rheumatoid Arthritis: A Cost-Effectiveness Analysis in the UK
}

\author{
Alex Diamantopoulos $\cdot$ A. Finckh $\cdot$ T. Huizinga $\cdot$ \\ D. K. Sungher $\cdot$ L. Sawyer $\cdot$ D. Neto $\cdot$ \\ F. Dejonckheere
}

Published online: 23 May 2014

(C) The Author(s) 2014. This article is published with open access at Springerlink.com

\begin{abstract}
Background Since receiving a positive recommendation in England, Wales and Scotland, tocilizumab (TCZ) is one of the options available to clinicians for the treatment of rheumatoid arthritis (RA) patients in the UK.

Objective The objective of this study was to evaluate the cost effectiveness of adding TCZ to the current treatment sequence of RA patients from a UK payer's perspective over a patient lifetime horizon.
\end{abstract}

Electronic supplementary material The online version of this article (doi:10.1007/s40273-014-0165-7) contains supplementary material, which is available to authorized users.

A. Diamantopoulos $(\bowtie) \cdot$ D. K. Sungher · L. Sawyer Symmetron Limited, Kinetic Centre, Theobald Street, Elstree, London WD6 4PJ, UK

e-mail: alexd@symmetron.net

\author{
A. Finckh · D. Neto \\ Rheumatology, University Hospitals of Geneva, Geneva, \\ Switzerland \\ T. Huizinga \\ Rheumatology, Leiden University Medical Center, Leiden, \\ The Netherlands \\ F. Dejonckheere \\ F. Hoffmann-La Roche, Basel, Switzerland
}

Methods An individual sampling model was developed to synthesise all clinical and economic inputs. Two scenarios were explored separately: patients contraindicated to methotrexate (MTX) and those MTX tolerant. For each scenario, the analysis compared three strategies. The standard of care (SoC) strategy included a sequence of the most commonly prescribed biologics; the other two comparator strategies considered the addition of TCZ to SoC at first line and second line. Patient characteristics were representative of UK patients. Treatment efficacy and qualityof-life evidence were synthesised from clinical trials and secondary sources. An analysis of a patient registry informed the model parameters regarding treatment discontinuation. The safety profile of all treatments in a given strategy was based on a network meta-analysis and literature review. Resource utilisation, treatment acquisition, administration, monitoring and adverse event treatment costs were considered. All costs reflect 2012 prices. Uncertainty in model parameters was explored by one-way and probabilistic sensitivity analysis.

Results In the MTX-contraindicated population, if TCZ was added to the $\mathrm{SoC}$ in first line, the estimated incremental cost-effectiveness ratio (ICER) was $£ 7,300$ per quality-adjusted life-year (QALY) gained; if added in second line, the estimated ICER was $£ 11,400$ per QALY. In the MTX-tolerant population, the estimated costs and QALYs of the TCZ strategy were similar to those of the SoC strategy. Sensitivity analysis showed that parameters that affect the treatment cost (such as patient weight) can have a noticeable impact on the overall cost-effectiveness results. The majority of the other sensitivity analyses resulted in modest changes to the ICER.

Conclusion For the treatment of RA in MTX-tolerant and contraindicated patients, the addition of TCZ to the SoC was estimated to be a cost-effective strategy. 


\section{Key Points for Decision Makers}

In methotrexate (MTX)-tolerant patients, the addition of tocilizumab (TCZ) to the standard biologic, disease-modifying anti-rheumatic drug, rheumatoid arthritis treatment sequence in the UK was associated with similar costs and a modest improvement in quality of life per patient.

In patients contraindicated to MTX, for whom there are fewer available treatments, the estimated qualityof-life benefit was more pronounced.

Overall, the addition of TCZ was estimated to be a cost-effective strategy, with a lower incremental cost-effectiveness ratio if used at first compared with second line.

\section{Introduction}

Rheumatoid arthritis (RA) is a chronic, progressive and disabling inflammatory condition typically causing symmetrical chronic arthritis characterised by joint pain, stiffness and swelling. It affects approximately $0.5-1 \%$ of the UK population and affects nearly three times as many women as men [1]. RA is associated with increased mortality, attributable at least in part to a higher risk of ischaemic heart disease as well to other factors, including infections related to co-morbidities, other systemic manifestations of the disease and immunosuppressive therapy [2-4].

Counting its direct, indirect and work-related disability costs, RA is estimated to cost the UK economy between $£ 3.8$ and $£ 4.75$ billion annually [5]. In early RA, these costs are driven by indirect costs, including the paid employment forgone by informal caregivers [6,7]. As RA progresses and pain, discomfort and physical impairment worsen, healthcare utilisation and medication costs become the principal contributors to overall cost [8].

In the absence of a curative treatment for RA, the focus of RA treatment is currently the prevention or control of joint damage, minimisation of loss of function and potential disability, avoidance of pain and improvement of quality of life $(\mathrm{QoL})$. Certain drugs such as glucocorticoids and non-steroidal anti-inflammatory drugs (NSAIDs) are effective in controlling RA symptoms; however, diseasemodifying anti-rheumatic drugs (DMARDs), alone or in combination, are the mainstay of RA management, and are used to slow progression of disease and improve function. They are divided into two categories: synthetic DMARDs
(sDMARDs)_including methotrexate (MTX), leflunomide, sulfasalazine, azathioprine, ciclosporin and hydroxychloroquine - and biologic DMARDs (bDMARDs) including abatacept, adalimumab, certolizumab, etanercept, golimumab, infliximab, rituximab and tocilizumab (TCZ). bDMARDs are licensed for the treatment of RA, but their use in the UK is currently restricted to patients who have failed to respond to (or tolerate) at least two sDMARDs.

An important clinical subgroup encompasses those patients in whom bDMARDs cannot be given in combination with MTX [9]. Therefore, this analysis focuses on both combination treatment as well as biologic monotherapy.

Tocilizumab is a humanised monoclonal antibody against the interleukin- 6 receptor. It is currently licensed for the treatment of RA and juvenile idiopathic arthritis (systemic juvenile idiopathic arthritis and polyarticular juvenile idiopathic arthritis) in combination with MTX, or as monotherapy in the case of intolerance to MTX or where continued treatment with MTX is inappropriate. A positive recommendation from the National Institute for Health and Care Excellence (NICE) and Scottish Medicines Consortium (SMC) in 2010 led to TCZ being reimbursed by the National Health Service (NHS) in the UK $[10,11]$.

The objective of this cost-effectiveness analysis was to determine whether the addition of TCZ at the beginning of a treatment pathway is cost effective in the treatment of moderate to severe RA.

\section{Methods}

\subsection{Model Overview}

The design of the economic analysis followed guidelines set by the Outcome Measures in Rheumatoid Arthritis Clinical Trials (OMERACT) Economics Working Group [12], and the structure was designed to closely represent clinical practice.

An individual sampling model, designed in MS Excel ${ }^{\circledR}$, was used to analyse transitions of patients through a sequence of treatments and, by maintaining patient history, translate this into economic and QoL outcomes.

The analysis compared the cost effectiveness of different strategies containing a sequence of biologic therapies licensed for use in patients with moderate to severe RA who had had an inadequate response (IR) to one or more sDMARDs. Two scenarios were explored separately: patients contraindicated to MTX and those who were MTX tolerant.

We defined the current standard of care (SoC) as a sequence of bDMARDs. In the MTX-tolerant population, the first treatment was an anti-tumour necrosis factor alpha 
Table 1 Strategies for MTXcontraindicated population

MTX methotrexate, $S o C$ standard of care, $T C Z$ tocilizumab

Table 2 Strategies for MTXtolerant population (biologics + MTX)

\begin{tabular}{llll}
\hline Line of treatment & Monotherapy SoC strategy & TCZ 1st line (strategy A) & TCZ 2nd line (strategy B) \\
\hline 1st & Certolizumab pegol & TCZ & Certolizumab pegol \\
2nd & Etanercept & Certolizumab pegol & TCZ \\
3rd & Adalimumab & Etanercept & Etanercept \\
4th & Palliative care & Adalimumab & Adalimumab \\
5th & & Palliative care & Palliative care \\
\hline & & & \\
\hline Line of & Combination therapy & TCZ 1st line & TCZ 2nd line \\
treatment & SoC strategy & (strategy C) & (strategy D) \\
\hline 1st & Certolizumab pegol & TCZ & Certolizumab pegol \\
2nd & Rituximab & Certolizumab pegol & TCZ \\
3rd & Etanercept & Rituximab & Rituximab \\
4th & Abatacept & Etanercept & Etanercept \\
5th & Adalimumab & Abatacept & Abatacept \\
6th & Infliximab & Adalimumab & Adalimumab \\
7th & Palliative care & Infliximab & Infliximab \\
8th & & Palliative care & Palliative care \\
\hline
\end{tabular}

Table 3 Baseline characteristics of model cohort

\begin{tabular}{lll}
\hline Characteristics & Model population & Source \\
\hline Female & $77 \%$ & {$[14]$} \\
Starting age & 58 years & \\
Starting HAQ score & 2 & \\
Starting VAS pain score & 75 & \\
Weight & $70 \mathrm{~kg}$ & Assumption \\
\hline
\end{tabular}

$H A Q$ Health Assessment Questionnaire, VAS Visual Analogue Scale

(aTNF) drug; certolizumab pegol has the lowest cost and therefore we assumed it would be offered first. Rituximab was offered following IR to the first aTNF, in accordance with the NICE guidance [13]. Thereafter, bDMARDs were ranked on the basis of their annual cost-assuming the one with the lowest cost would be used first. The MTX-contraindicated population model followed a similar ranking, including only treatments with a licence for monotherapy. Alternative sequences for the SoC were explored during the development of the model; the current one was demonstrated to be the most conservative.

We considered the addition of TCZ to the current SoC strategy, and explored its effect at first line and second line (see Tables 1 and 2).

The characteristics of the model cohort were based on data from the British Society for Rheumatology Biologics Register (BSRBR) [14]; they are consistent with the drug licence and are representative of the UK RA population (see Table 3). The average patient weight was assumed to be $70 \mathrm{~kg}[13,15]$. The analysis time horizon was patient lifetime.
American College of Rheumatology (ACR) response probabilities were used to measure response to treatment as these are readily available from the randomised controlled trial (RCT) evidence. The model used a 6-month cycle length, which is in line with the timing of most RCT outcomes included in the analysis. Disease severity was represented by changes in Health Assessment Questionnaire (HAQ) score and Visual Analogue Scale (VAS) pain score, two surrogate health outcomes which can be translated to utility scores and ultimately to quality-adjusted life-years (QALYs). Once patients exhausted all treatments in the sequence, they moved into palliative care, where they remained until death. Patients could transition to death on the basis of a mortality risk adjusted for RA [16]. The possible transitions and the structure of the model are illustrated in Fig. 1.

The analysis took a UK NHS perspective, with costs expressed in 2011/2012 pound sterling and health benefits measured in QALYs. Both costs and benefits were discounted at an annual rate of $3.5 \%$, and half-cycle correction was applied. The performance of alternative treatment sequences was estimated using incremental cost-effectiveness ratios (ICERs), defined as the added cost of a given strategy divided by its added benefit compared with the next most expensive strategy.

\subsection{Treatment Efficacy}

Efficacy in the model was determined by response to treatment, which was subsequently translated to a drop in HAQ score and change in VAS pain. Responders were categorised according to their level of ACR response- 


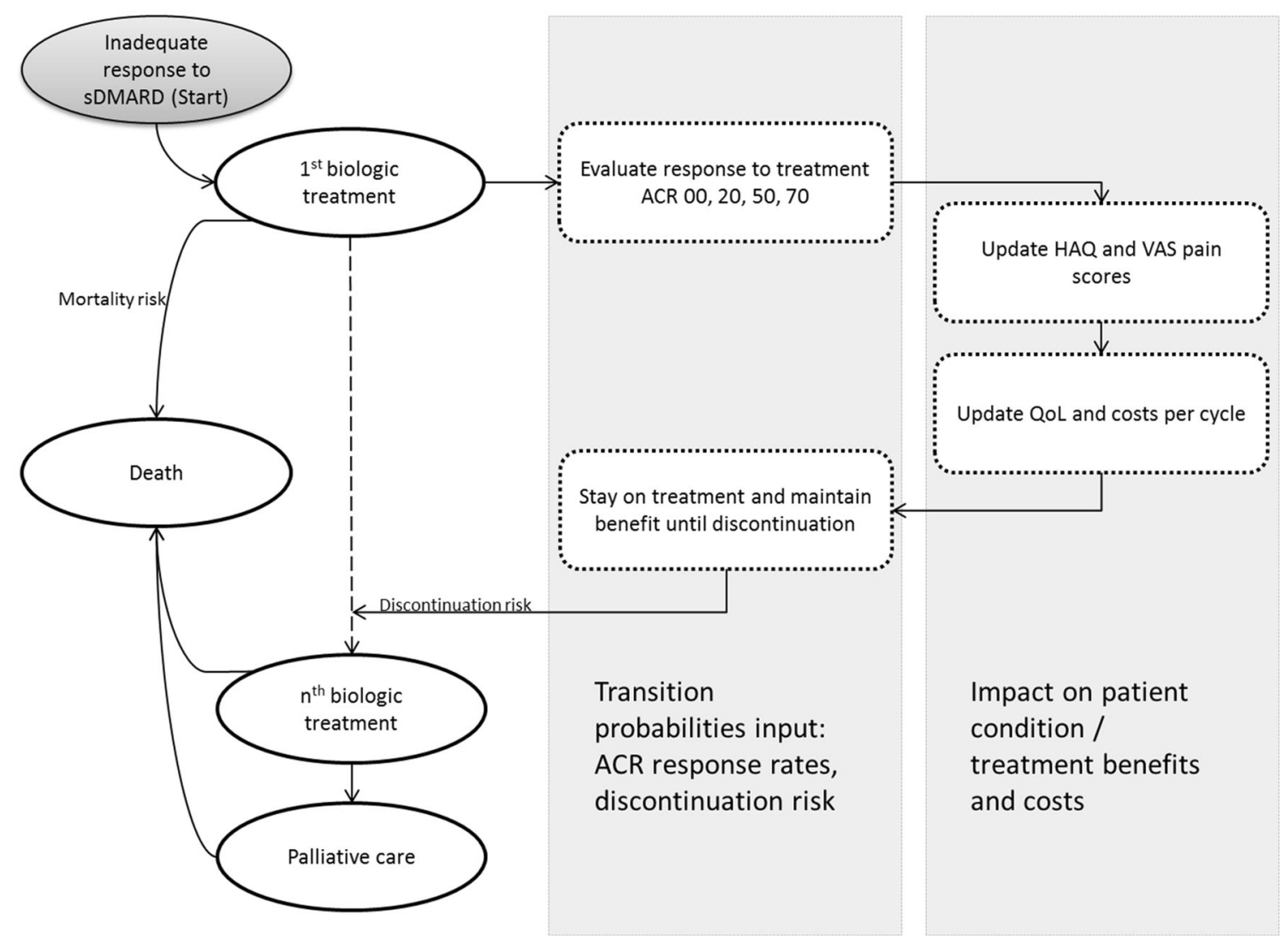

Fig. 1 Model structure, health states and transitions. ACR American College of Rheumatology, HAQ Health Assessment Questionnaire, $Q o L$ quality of life, $s D M A R D$ synthetic disease-modifying anti-rheumatic drugs, VAS Visual Analogue Scale

ACR00 (no response), ACR20, ACR50 and ACR70. The proportion of patients achieving each level of response differed by treatment and was derived through a network meta-analysis (NMA) of RCT data (see Table 4) [17, 18]. MEDLINE $^{\circledR}$ and EMBASE $^{\circledR}$ databases were searched simultaneously for articles published in English, from 1990 to October 2011, using a predefined search strategy. To allow comparison with the recent TCZ studies, the literature review focused on studies that included only patients with sDMARD inadequate response (sDMARD-IR). Twenty-two relevant studies were identified with ACR response rates at 24 weeks. Despite some variation in the patient characteristics across the identified studies (i.e. duration of disease), there were no observed systematic differences, indicating the feasibility of the indirect comparison. Because of limited data identified for aTNFs used in monotherapy, response data for all aTNF treatments were pooled, assuming exchangeability of the efficacy profile for those agents. An NMA was conducted (Bayesian framework) to synthesise the identified evidence and to obtain effect estimates for the bDMARDs. The probability of ACR response at 24 weeks for the bDMARDs is presented in Table 4. Rituximab was used in the model in an aTNF-IR position (in line with its label). Data from a study on aTNF-IR patients was used for the ACR response probability with rituximab [18].

Response to treatment was assumed to impact disease severity and pain, as measured by HAQ and VAS pain score, respectively. The impact of response to treatment on HAQ and VAS pain score was assumed to be treatment independent. Patient-level data from three phase III clinical trials were pooled and analysed to determine the relationship of ACR response with HAQ (2,204 observations) and VAS pain score (2,342 observations) (details presented in Appendix A in the Electronic Supplementary Material) [19-21]. These studies were selected because their respective patient characteristics were similar to those assumed in the economic model and patient-level data were available for analysis during the model development. Results suggest a negative correlation between both ACR response and HAQ score and ACR response and VAS pain score; that is, the higher the observed ACR response the greater the drop in HAQ and VAS pain scores (see Table 4).

The relationship between ACR response, HAQ and VAS pain was assumed to be the same across all treatments. A patient's disease was assumed to remain stable whilst on biologic treatment and progress whilst on palliative care, 
Table 4 Key model parameters and assumptions

\begin{tabular}{|c|c|c|}
\hline Variable & Value & Description (source) \\
\hline \multicolumn{3}{|l|}{ ACR response $(\%)$, probability of ACR20/50/70 } \\
\hline \multicolumn{3}{|l|}{ Combination therapy } \\
\hline $\mathrm{aTNF}+\mathrm{MTX}$ & $72 / 52 / 17$ & \multirow[t]{5}{*}{ Generated from NMA [17] } \\
\hline $\mathrm{TCZ}+\mathrm{MTX}$ & $67 / 51 / 23$ & \\
\hline Abatacept + MTX & $64 / 42 / 13$ & \\
\hline Infliximab & $72 / 52 / 17$ & \\
\hline Palliative care (placebo rates) & $33 / 16 / 3$ & \\
\hline Rituximab + MTX & $51 / 27 / 12$ & Unadjusted rates from Cohen et al. [18] \\
\hline \multicolumn{3}{|l|}{ Monotherapy (MTX contraindicated) } \\
\hline aTNF & $50 / 27 / 12$ & \multirow[t]{3}{*}{ Generated from NMA [17] } \\
\hline TCZ & $66 / 46 / 24$ & \\
\hline Palliative care (placebo rates) & $17 / 6 / 1$ & \\
\hline \multicolumn{3}{|c|}{ Average decrease in HAQ by ACR response category (SE) } \\
\hline$<20$ & $0.14(0.02)$ & \multirow{4}{*}{$\begin{array}{l}\text { Post hoc analysis [19-21]. See Appendix A in } \\
\text { the Electronic Supplementary Material }\end{array}$} \\
\hline$\geq 20$ and $<50$ & $0.46(0.02)$ & \\
\hline$\geq 50$ and $<70$ & $0.68(0.03)$ & \\
\hline$\geq 70$ & $0.90(0.03)$ & \\
\hline \multicolumn{3}{|c|}{ Average decrease in VAS pain score by ACR category (SE) } \\
\hline$<20$ & $4.02(0.79)$ & \multirow{4}{*}{$\begin{array}{l}\text { Post-hoc analysis [19-21]. See Appendix A } \\
\text { in the Electronic Supplementary Material }\end{array}$} \\
\hline$\geq 20$ and $<50$ & $23.97(0.86)$ & \\
\hline$\geq 50$ and $<70$ & $36.00(0.95)$ & \\
\hline$\geq 70$ & $45.96(1.26)$ & \\
\hline Average VAS pain score in palliative care & $55(1.28)$ & Clinical expert opinion \\
\hline \multirow{2}{*}{\multicolumn{3}{|c|}{$\begin{array}{l}\text { Probability of discontinuation from treatment } \\
\text { bDMARD-naïve (loglogistic model) }\end{array}$}} \\
\hline & & \\
\hline Scale & 0.76262 & Based on analysis of SCQM-RA data \\
\hline Intercept & 3.33 & \multirow{2}{*}{$\begin{array}{l}\text { See variance covariance matrix in Appendix } \\
\text { B (Electronic Supplementary material) }\end{array}$} \\
\hline Responder & 0.582 & \\
\hline \multicolumn{3}{|l|}{ bDMARD-IR (Lognormal model) } \\
\hline Scale & 1.44484 & \multirow{3}{*}{$\begin{array}{l}\text { Based on analysis of SCQM-RA data. See } \\
\text { variance covariance matrix in Appendix B } \\
\text { (Electronic Supplementary Material) }\end{array}$} \\
\hline Intercept & 3.206 & \\
\hline Responder & 0.545 & \\
\hline \multicolumn{3}{|l|}{ Probability of serious infection (SE) } \\
\hline Certolizumab pegol & $0.113(0.074)$ & [26] \\
\hline bDMARD (excluding certolizumab pegol) & $0.035(0.0048)$ & {$[26]$} \\
\hline \multicolumn{3}{|c|}{ Average 6-monthly HAQ score decrease on treatment (SE) } \\
\hline bDMARDs & $0(0.0057)$ & [22] \\
\hline sDMARDs & $0.0225(0.0019)$ & [22] \\
\hline Palliative care & 0.03 & [22] \\
\hline \multicolumn{3}{|c|}{ Utility multiplier for AEs (synthesised using methods from Ara and Brazier [34]) } \\
\hline bDMARDs & 0.9858 & [33] \\
\hline Palliative care/MTX-combination model & 0.9700 & $\begin{array}{l}\text { Appendix C and D in the Electronic } \\
\text { Supplementary Material }\end{array}$ \\
\hline Palliative care/MTX contraindicated & 0.9717 & $\begin{array}{l}\text { Appendix C and D in the Electronic } \\
\text { Supplementary Material }\end{array}$ \\
\hline
\end{tabular}

ACR American College of Rheumatology, AEs adverse events, aTNF anti-tumour necrosis factor alpha, $b D M A R D$ biologic disease-modifying anti-rheumatic drug, $b D M A R D$-IR biologic disease-modifying anti-rheumatic drug inadequate response, $H A Q$ Health Assessment Questionnaire, $M T X$ methotrexate, NMA network meta-analysis, $S E$ standard error, $S D M A R D$ synthetic disease-modifying anti-rheumatic drug, TCZ tocilizumab, VAS Visual Analogue Scale 
an approach taken by previous evidence reimbursement submissions in the UK $[10,11]$ and a recent NICE multiple technology appraisal [22] (see Table 4). The level of VAS pain for patients in palliative care was assumed to be 55 , on the basis of clinical expert opinion (AF, TH).

\subsection{Treatment Discontinuation}

Since patients were assumed to try all drugs in a given strategy, treatment discontinuation became an important driver of the model outcomes. An analysis of a large biologics data registry ( $n=1,464$ without rituximab sample) in Europe (Swiss Clinical Quality Management in Rheumatic Diseases [SCQM-RA]) showed that discontinuation can be response related. Since the SCQM-RA data set did not have ACR response evidence for many patients, we used European League Against Rheumatism (EULAR) response data as a proxy for this input [23]. A range of parametric survival models (Weibull, exponential, loglogistic, lognormal) were explored. We selected the best fitting model based on Akaike Information Criteria (AIC) and visual inspection of the resulting curves against the SCQM-RA Kaplan-Meier curves and UK observational data [24]. The loglogistic model had the best fit for bDMARD-naive patients, and the lognormal model for the bDMARD-IR patients. These were used as a base case while alternative models were tested in the sensitivity analysis (details of the treatment discontinuation analysis are presented in Appendix B in the Electronic Supplementary Material).

Little evidence exists on the long-term sustained benefit of treatment after patients withdraw because of a lack of efficacy. Some evidence suggests that a "rebound" occurs when therapy is withdrawn [25]. In our analysis, we assumed that HAQ worsening was equal to the initial HAQ improvement and that it occurred immediately at the point of withdrawal. That is, patients who withdrew because of a lack of efficacy immediately returned to their starting HAQ score, until they began their next treatment.

\subsection{Treatment Safety}

The safety of biologic treatments was derived from a recent systematic review and NMA [26]. The pooled results for all biologic treatments indicated that the odds of serious infection (defined as those associated with death, hospitalisation and use of intravenous antibiotics) were significantly greater than for control [odds ratio 1.37, $95 \%$ confidence interval (CI) 1.04-1.82]. Singh and colleagues [26] also presented results for each individual biologic therapy, among which only the odds ratio for certolizumab pegol reached statistical significance $(4.75,95 \%$ CI 1.52-18.65). In order to reflect this difference, the probability of serious infection for certolizumab pegol was derived from its individual odds ratio (4.75) and for all other biologics from the generic odds ratio (1.37). The resulting absolute risks, which were applied at every cycle whilst on a given treatment, are shown in Table 4.

The safety of drugs typically used as part of palliative care was also reviewed. A survey of clinical experts indicated that most patients who have failed all previous biologic treatments would be on a mixture of therapies including glucocorticoids and sDMARDs for disease management, selective and non-selective NSAIDs for symptom control, and opioids and analgesics for pain relief. Reviews of those treatments indicated that the most frequent adverse events (AEs) include dyspepsia, nausea, constipation, infections, headaches, dizziness, skin rash/ allergy and alopecia $[19,27-31]$. The incidence of these events was combined with associated costs and utilities for inclusion in the economic model (details of the palliative care survey are presented in Appendix $\mathrm{C}$ in the Electronic Supplementary Material).

\subsection{Health-Related Quality of Life}

Change in HAQ, VAS pain and the associated utility gain were used in the model to determine the impact of treatment on overall health. Estimates of utility gain were calculated using a regression model for mapping HAQ scores to the EuroQol 5-Dimensions (EQ-5D) score [32]. This model represents an improvement to previous mapping algorithms in that it accommodates not only the ceiling effect and the gap in the data but also the observed multimodality in EQ-5D. It also considers the effect of VAS pain, which is one of the most heavily weighted items in the EQ-5D. The EQ-5D score for each simulated individual was estimated based on the Alava model, the simulated HAQ score which is linked to ACR response, VAS pain scores from the clinical trials (Table 4), and patient age in each cycle.

For the disutility from serious AEs while on a bDMARD, we reviewed six of the largest studies identified from the Cochrane safety review [26]. We found that the most common and significant serious infection was pneumonia [19, 27-31]. A utility for pneumonia was identified in the literature-0.21 [33]; it was adjusted for the expected duration of the event (7 days) and the baseline age and gender of the Sisk et al. [33] cohort. The resulting utility multiplier (0.9858) was combined with the incidence of serious infections and applied to the baseline utility derived from the Alava model.

Disutility for AEs associated with palliative care was identified through a targeted review of published economic evaluations, and multipliers were derived in the same fashion (details in Appendix D in the Electronic 
Supplementary Material). Utility multipliers were then combined with the incidence of each $\mathrm{AE}$ to estimate a weighted average utility multiplier for palliative care; note that this is slightly different for patients receiving MTX and those for whom MTX is contraindicated (Table 4).

\subsection{Treatment-Related Costs}

The treatment cost for each drug was sourced from the British National Formulary [35], and the dose schedules for each treatment were taken from the electronic Medicines Compendium (eMC) website [36] (see Table 5). For weight-based dosing, the average patient was assumed to weigh $70 \mathrm{~kg}$. A patient access scheme (PAS) for certolizumab pegol was considered in the calculations; the manufacturer provides the first 12 weeks (ten pre-loaded 200-mg syringes) free of charge to all patients starting treatment.

Ten per cent of subcutaneous injection treatment was assumed to be administered by a district nurse. Monitoring patients who receive subcutaneously injected medicines was assumed to involve an outpatient visit or a general practitioner visit and certain examinations and tests, such as full blood count, erythrocyte sedimentation rate, C-reactive protein, liver function test, chest X-ray, urea, electrolytes and creatinine. The resource use assumed in this analysis was consistent with previous evidence reimbursement submissions $[10,11]$. The cost of administering and monitoring intravenous drugs was assumed to be $£ 171.33$ per infusion [37].

The drug cost for palliative care was based on a weighted average of treatments (glucocorticoids, sDMARDs, selective and non-selective NSAIDs, opioids). No costs were assumed for administering palliative care, but monitoring costs were assumed to be high, driven by a greater number of primary and secondary care visits and blood tests.

\subsection{Adverse Event-Related Costs}

The cost for pneumonia was sourced from the NHS reference costs [38]. The costs of other AEs associated with drugs used in palliative care included those of dyspepsia and constipation. No costs were reported for experiencing nausea, hypertension, headaches, dizziness, skin rash/ allergy or alopecia. These were synthesised with incidence figures and relative usage of different drugs from the expert survey to arrive at a set of values for palliative care (see Table 5).

\subsection{Disease-Related Costs}

In addition to treatments received and the monitoring included therein, many patients were assumed to require inpatient care associated with their RA. The model assumed six different bands of HAQ score to reflect mild,
Table 5 Treatment, AE and disease-related costs

$A E$ adverse event, $H A Q$ Health Assessment Questionnaire, MTX methotrexate, TCZ tocilizumab

\begin{tabular}{ll}
\hline Variable & Value \\
\hline Combination therapy (total cost first 6 months/subsequent cycles) & \\
Adalimumab (40 mg every 2 weeks) + MTX & $£ 6,324 / £ 5,261$ \\
Certolizumab pegol (200 mg every 2 weeks) + MTX & $£ 4,070 / £ 5,331$ \\
Etanercept (50 mg every week) + MTX & $£ 6,429 / £ 5,366$ \\
TCZ ( 8 mg/kg every 4 weeks) + MTX & $£ 4,776 / £ 4,776$ \\
Abatacept (750 mg every 4 weeks) + MTX & $£ 7,015 / £ 7,015$ \\
Infliximab ( 3 mg/kg every 8 weeks $)+$ MTX & $£ 3,425 / £ 3,425$ \\
Rituximab $(2 \times 1,000$ mg per 6 months $)+$ MTX & $£ 3,840 / £ 3,840$ \\
Palliative care & $£ 2,589 / £ 1,287$ \\
AE-related costs & \\
Serious infection & $£ 2,047$ \\
Combined AEs for palliative care & \\
Combination therapy model & $£ 168$ \\
Monotherapy model & $£ 141$ \\
Patient condition-related costs & \\
HAQ $<0.5$ & $£ 62(0.26)$ \\
HAQ $\geq 0.6$ and $<1.0$ & $£ 31(0.13)$ \\
HAQ $\geq 1.0$ and $<1.5$ & $£ 122(0.51)$ \\
HAQ $\geq 1.5$ and $<2.0$ & $£ 173(0.72)$ \\
HAQ $\geq 2.0$ and $<2.6$ & $£ 446(1.86)$ \\
HAQ $\geq 2.6$ and $<3.0$ & $£ 998(4.16)$ \\
\hline
\end{tabular}


moderate and severe disease $[39,40]$. Data from the Norfolk Arthritis Register (NOAR) in the UK were analysed such that mean days in hospital over a 12-month period could be estimated for each HAQ score category. Based on a cost of $£ 240$ per inpatient day, each HAQ score category was assigned a cost (see Table 5).

\subsection{Sensitivity Analysis and Parameter Uncertainty}

One-way sensitivity analysis (OWSA) was performed to identify the model drivers and the magnitude of their impact on the model results. In order to explore uncertainty around the model's input, probabilistic sensitivity analysis (PSA) was also performed (1,000 samples).

\section{Results}

Aggregate results were based on 3,000 simulations of hypothetical patient pathways. Table 6 displays the costeffectiveness results for the monotherapy strategies. The estimated total cost for the SoC strategy was $£ 139,000$; the total QALYs accrued were 8.0162 over an average 21.5 years in the model. The addition of TCZ to the SoC after sDMARD-IR increased the estimated total cost to $£ 143,000$ and produced 8.4987 QALYs. In the TCZ strategy, time on biologic treatment was extended by an average of 2.5 years compared with the SoC strategy. The ICER was estimated to be $£ 7,300$ per QALY gained. If TCZ was added in the sequence in later positions (e.g. second line), the costs and QALYs increased. The ICER was estimated to be $£ 11,400$ per QALY gained.

Table 7 presents the cost-effectiveness results for the combination treatment strategies. The three strategies were similar in terms of total costs and total QALYs gained. The number of patients receiving palliative care was reduced substantially across all comparator strategies, on average by 3 and $6 \%$ of simulated individuals on TCZ or SoC strategy, respectively. In the SoC strategy, patients were estimated to stay on biologics for 21 years. In the strategy by which TCZ was offered first after sDMARD-IR, patients

Table 6 Base-case cost-effectiveness results for monotherapy strategies

\begin{tabular}{llllr}
\hline & $\begin{array}{l}\text { Total drug } \\
\text { costs }\end{array}$ & Total costs & $\begin{array}{l}\text { Total } \\
\text { QALYs }\end{array}$ & ICER \\
\hline SoC & $£ 130,736.86$ & $£ 139,008.09$ & 8.0162 & \\
TCZ 1st line & $£ 136,157.48$ & $£ 142,525.23$ & 8.4987 & $£ 7,289.63$ \\
TCZ 2nd line & $£ 137,686.19$ & $£ 144,744.15$ & 8.5194 & $£ 11,400.26$ \\
\hline
\end{tabular}

ICER incremental cost-effectiveness ratio, $Q A L Y$ quality-adjusted life-year, $S o C$ standard of care, $T C Z$ tocilizumab stayed on biologics for almost the duration of the model (21.5 years on average).

The OWSA is presented in Tables 8 and 9. Respective changes to model parameters have the same impact in both the monotherapy and the MTX-combination analyses. Overall, the model was sensitive to changes in the TCZ treatment cost. Changes to the assumed patient weight affected the TCZ cost and consequently the final ICER. Given that the results between the two comparator strategies are similar in the combination model, the increase in cost changes the conclusion of the cost-effectiveness analysis; the TCZ strategy no longer produces cost-savings and the result is a high ICER.

Assumptions around palliative care did not have a substantial impact on the overall cost-effectiveness results; neither did changes to the assumptions on AEs of biologics or ACR response rates [9]. The choice of discontinuation risk (time dependent or constant) and the choice of QoL mapping model had a moderate effect on the model results.

Figures 2 and 3 present the results of the PSA with costeffectiveness acceptability curves for the monotherapy and the combination with MTX analyses. In both cases, the probability of cost effectiveness was estimated to be higher when TCZ was added first in the treatment sequence. Details of the parameters, ranges and distributions assumed for the PSA are presented in Appendix E in the Electronic Supplementary Material.

\section{Discussion}

To our knowledge, this is the first analysis investigating the cost effectiveness of adding TCZ to SoC, compared as monotherapy or in combination with MTX, in treating adults with moderate to severe RA in the UK. The analysis was based on the reimbursement application to NICE in the UK; all assumptions follow the NICE reference case [41].

Our economic evaluation synthesised evidence of patient characteristics of a UK population [14], evidence from an NMA of randomised clinical trials for drug efficacy [17], registry data analysis for discontinuation (SCQM-RA), and cost estimates from the NHS UK perspective, with a PAS where applicable. With regard to QoL estimates, as noted in the "Methods" section, the analysis used a mapping model that the authors here consider an improvement on previous algorithms [25, 42-44].

In both populations (monotherapy and MTX combination), the addition of TCZ to the current treatment sequence in the UK appeared to be a cost-effective strategy. However, its impact in QALYs gained was more pronounced in the monotherapy than in the combination model. Part of this was due to the additional benefits in QALYs driven by TCZ efficacy, and another part was due to the displacing of 
Table 7 Base-case cost-effectiveness results for combination therapy strategies

\begin{tabular}{lllll}
\hline & Total drug costs & Total costs & Total QALYs & ICER \\
\hline SoC & $£ 144,555.66$ & $£ 150,665.03$ & 8.8609 & Dominated by TCZ 1st line \\
TCZ 1st line & $£ 142,337.63$ & $£ 147,640.97$ & 8.9050 & Dominant \\
TCZ 2nd line & $£ 144,129.33$ & $£ 150,127.31$ & 8.8983 & Dominated by TCZ 1st line \\
\hline
\end{tabular}

ICER incremental cost-effectiveness ratio, $Q A L Y$ quality-adjusted life-year, $S o C$ standard of care, TCZ tocilizumab

Table 8 One-way sensitivity analysis of cost-effectiveness results for monotherapy strategies

\begin{tabular}{|c|c|c|c|c|c|c|}
\hline \multirow[t]{2}{*}{ Scenario } & \multirow[t]{2}{*}{ Description } & \multicolumn{2}{|l|}{$\mathrm{SoC}$} & \multicolumn{2}{|l|}{ TCZ strategy } & \multirow[t]{2}{*}{ ICER } \\
\hline & & Total cost & Total QALYs & Total cost & Total QALYs & \\
\hline Base case & Base case- $-\mathrm{TCZ}$ at 1 st line & $£ 139,008.09$ & 8.016 & $£ 142,525.23$ & 8.499 & $£ 7,289.63$ \\
\hline Scenario 1 & $\begin{array}{l}\text { Using Weibull model for discontinuation } \\
\text { (SCQM-RA) }\end{array}$ & $£ 134,751.18$ & 7.834 & $£ 140,255.64$ & 8.395 & $£ 9,816.87$ \\
\hline Scenario 2 & $\begin{array}{l}\text { Using constant discontinuation risks } \\
\text { (SCQM-RA) }\end{array}$ & $£ 125,168.26$ & 7.334 & $£ 133,266.16$ & 8.003 & $£ 12,095.97$ \\
\hline Scenario 3 & Assume no cost for palliative care & $£ 137,002.34$ & 8.016 & $£ 141,508.25$ & 8.499 & $£ 9,338.92$ \\
\hline Scenario 4 & $\begin{array}{l}\text { Palliative care cost equals the average cost } \\
\text { of all biologics }\end{array}$ & $£ 160,654.65$ & 8.016 & $£ 153,500.87$ & 8.499 & TCZ dominates \\
\hline Scenario 5 & $\begin{array}{l}\text { Assume no disutility for palliative care: baseline } \\
\text { HAQ score is } 0\end{array}$ & $£ 138,815.92$ & 8.351 & $£ 142,453.87$ & 8.658 & $£ 11,843.09$ \\
\hline Scenario 6 & Patients' weight at baseline is $60 \mathrm{~kg}$ & $£ 139,008.09$ & 8.016 & $£ 136,191.31$ & 8.499 & TCZ dominates \\
\hline Scenario 7 & Patients' weight at baseline is $80 \mathrm{~kg}$ & $£ 139,008.09$ & 8.016 & $£ 148,859.16$ & 8.499 & $£ 20,417.30$ \\
\hline Scenario 8 & bDMARDs assume no AE disutility or cost & $£ 135,515.90$ & 8.096 & $£ 139,530.04$ & 8.539 & $£ 9,057.90$ \\
\hline Scenario 9 & $\begin{array}{l}\text { Use QoL equation from Bansback et al. [43]; } \\
\text { QoL }=0.76-0.28 \times \mathrm{HAQ}+0.05 \times \text { female }\end{array}$ & $£ 139,008.09$ & 4.354 & $£ 142,525.23$ & 4.827 & $£ 7,426.08$ \\
\hline Scenario 10 & $\begin{array}{l}\text { Use QoL equation from MabThera analysis [42]; } \\
\text { QoL }=0.862-0.327 \times \text { HAQ }\end{array}$ & $£ 139,008.09$ & 4.085 & $£ 142,525.23$ & 4.625 & $£ 6,506.64$ \\
\hline Scenario 11 & $\begin{array}{l}\text { Use QoL equation from Ducournau et al. [44]; } \\
\text { QoL }=0.8229-0.1125 \times \text { HAQ }- \\
0.06874 \times \mathrm{HAQ}^{2}\end{array}$ & $£ 139,008.09$ & 5.731 & $£ 142,525.23$ & 6.339 & $£ 5,784.48$ \\
\hline Scenario 12 & ACR data from NICE Assessment Report [9] & $£ 139,681.68$ & 8.051 & $£ 143,248.67$ & 8.509 & $£ 7,782.19$ \\
\hline
\end{tabular}

$A C R$ American College of Rheumatology, $A E$ adverse event, bDMARDs biologic disease-modifying anti-rheumatic drugs, $H A Q$ Health Assessment Questionnaire, ICER incremental cost-effectiveness ratio, NICE National Institute of Health and Care Excellence, $Q A L Y$ qualityadjusted life-year, $Q o L$ quality of life, SCQM-RA Swiss Clinical Quality Management in Rheumatic Diseases, SoC standard of care, TCZ tocilizumab

time on palliative care. Since the latter is more likely to happen in the monotherapy model, where there are fewer treatment options for an RA patient, the impact is more pronounced there and less in the combination treatment model, where, on average, patients will spend significantly less time on palliative care, in the last months of their lives.

The assumptions on the risk of discontinuation were an important driver of the model, especially since we assumed that patients would try all treatments in a strategy. We ran a post hoc analysis of observational data to estimate the probability of discontinuation (assumed time dependent). It is a limitation of this study that we did not have access to a UK registry for this analysis, and therefore the input data could suffer from generalising from a Swiss cohort of patients. Nevertheless, when we compared the data with evidence from Hyrich et al. [14] and Soliman et al. [24] from the UK, the resulting risk was found to be similar. Another limitation regarding the SCQM-RA data analysis is that because of a very small number of ACR response data, we used EULAR response as a proxy.

Our model used the ACR response criteria to weigh the benefit of treatment (HAQ, VAS pain scores and QALYs). Ideally, the model could use Disease Activity Scores using 28 joints or EULAR response, in line with NICE decision rules. The use of ACR response criteria ensures a wider choice of studies that inform our NMA and, on balance we considered this to be more important for the economic model input.

With regard to the treatment cost calculations, after the addition of administration and monitoring costs, the 
Table 9 One-way sensitivity analysis of cost-effectiveness results for combination therapy strategies

\begin{tabular}{|c|c|c|c|c|c|c|}
\hline \multirow[t]{2}{*}{ Scenario } & \multirow[t]{2}{*}{ Description } & \multicolumn{2}{|l|}{$\mathrm{SoC}$} & \multicolumn{2}{|l|}{ TCZ strategy } & \multirow[t]{2}{*}{ ICER } \\
\hline & & Total cost & Total QALYs & Total cost & Total QALYs & \\
\hline Base case & Base case- $-\mathrm{TCZ}$ at 1 st line & $£ 150,665.03$ & 8.861 & $£ 147,640.97$ & 8.905 & TCZ dominates \\
\hline Scenario 1 & $\begin{array}{l}\text { Using Weibull model for discontinuation } \\
\text { (SCQM-RA) }\end{array}$ & $£ 151,349.35$ & 8.854 & $£ 148,061.14$ & 8.923 & TCZ dominates \\
\hline Scenario 2 & $\begin{array}{l}\text { Using constant discontinuation risks } \\
\text { (SCQM-RA) }\end{array}$ & $£ 148,216.33$ & 8.687 & $£ 146,987.86$ & 8.814 & TCZ dominates \\
\hline Scenario 3 & Assume no cost for palliative care & $£ 150,500.20$ & 8.861 & $£ 147,571.42$ & 8.905 & TCZ dominates \\
\hline Scenario 4 & $\begin{array}{l}\text { Palliative care cost equals the average } \\
\text { cost of all biologics }\end{array}$ & $£ 152,807.51$ & 8.861 & $£ 148,544.93$ & 8.905 & TCZ dominates \\
\hline Scenario 5 & $\begin{array}{l}\text { Assume no disutility for palliative care: } \\
\text { baseline HAQ score is } 0\end{array}$ & $£ 150,655.89$ & 8.888 & $£ 147,639.40$ & 8.916 & TCZ dominates \\
\hline Scenario 6 & Patients' weight at baseline is $60 \mathrm{~kg}$ & $£ 150,391.10$ & 8.861 & $£ 141,165.45$ & 8.905 & TCZ dominates \\
\hline Scenario 7 & Patients' weight at baseline is $80 \mathrm{~kg}$ & $£ 150,938.95$ & 8.861 & $£ 154,116.48$ & 8.905 & $£ 72,107.33$ \\
\hline Scenario 8 & bDMARDs assume no AE disutility or cost & $£ 146,780.20$ & 8.869 & $£ 144,432.09$ & 8.912 & TCZ dominates \\
\hline Scenario 9 & $\begin{array}{l}\text { Use QoL equation from Bansback et al. [43]; } \\
\text { QoL }=0.76-0.28 \times \text { HAQ }+0.05 \times \text { female }\end{array}$ & $£ 150,665.03$ & 5.250 & $£ 147,640.97$ & 5.326 & TCZ dominates \\
\hline Scenario 10 & $\begin{array}{l}\text { Use QoL equation from MabThera analysis [42]; } \\
\text { QoL }=0.862-0.327 \times \text { HAQ }\end{array}$ & $£ 150,665.03$ & 5.105 & $£ 147,640.97$ & 5.194 & TCZ dominates \\
\hline Scenario 11 & $\begin{array}{l}\text { Use QoL equation from Ducournau et al. [44]; } \\
\text { QoL }=0.8229-0.1125 \times \text { HAQ } \\
-0.06874 \times \mathrm{HAQ}^{2}\end{array}$ & $£ 150,665.03$ & 6.884 & $£ 147,640.97$ & 6.972 & TCZ dominates \\
\hline Scenario 12 & ACR data from NICE Assessment Report [9] & $£ 149,735.11$ & 8.912 & $£ 147,251.97$ & 8.935 & TCZ dominates \\
\hline
\end{tabular}

$A C R$ American College of Rheumatology, $A E$ adverse event, $b D M A R D s$ biologic disease-modifying anti-rheumatic drugs, $H A Q$ Health Assessment Questionnaire, ICER incremental cost-effectiveness ratio, NICE National Institute of Health and Care Excellence, $Q A L Y$ qualityadjusted life-year, $Q o L$ quality of life, SCQM-RA Swiss Clinical Quality Management in Rheumatic Diseases, SoC standard of care, TCZ tocilizumab

Fig. 2 Cost-effectiveness acceptability curve for monotherapy strategies. SoC standard of care, $T C Z$ tocilizumab

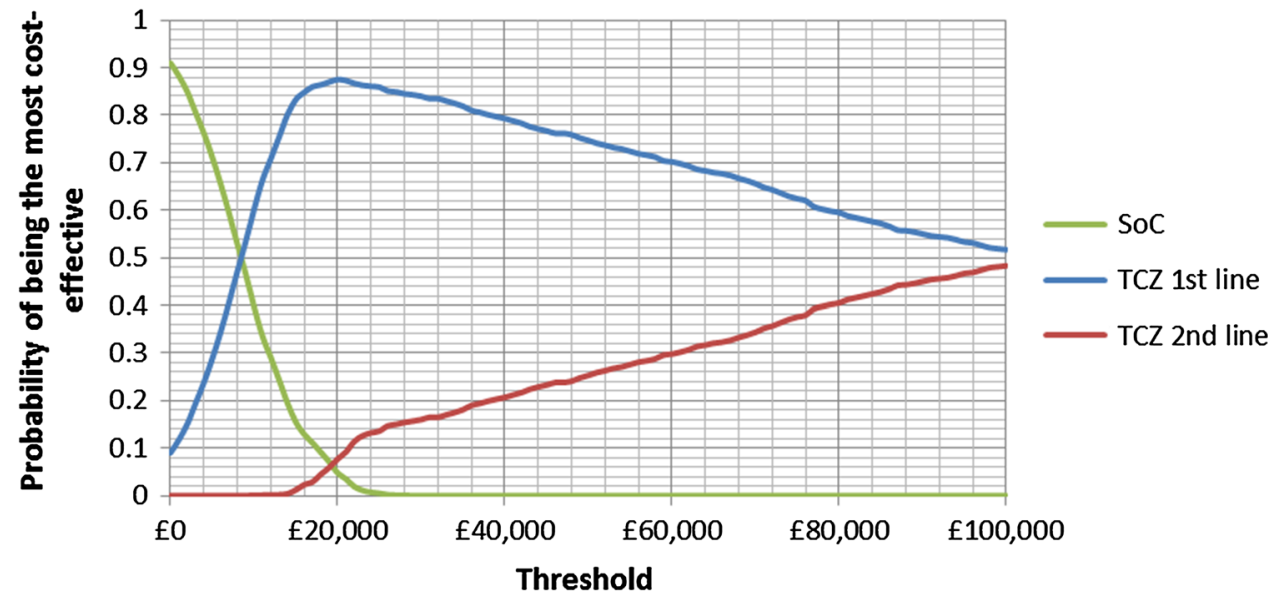

average total cost for a biologic was just over $£ 10,000$ per year. Since our comparison was one of treatment strategies, it was the cost of TCZ (solely based on patients' weight) that had the most impact on the cost-effectiveness resultsas shown from the sensitivity analysis.

The research conducted on the cost of palliative care (patient monitoring and safety) added to the completeness of the overall analysis, but had no notable impact on the model results; its overall cost was very low compared with the add-on bDMARD (TCZ). At the development phase of the palliative care survey, we anticipated greater convergence amongst the individual responses. However, we found large variation amongst responses, and this prohibited any statistical analysis or summarisation of the survey findings. This could be due to methodological issues: the type of questions, the format of the interview, the rounds of revision or clarifications, etc. It also appears likely that since clinicians are less and less likely to face those cases 
Fig. 3 Cost-effectiveness acceptability curve for combination treatment strategies. SoC standard of care, $T C Z$ tocilizumab

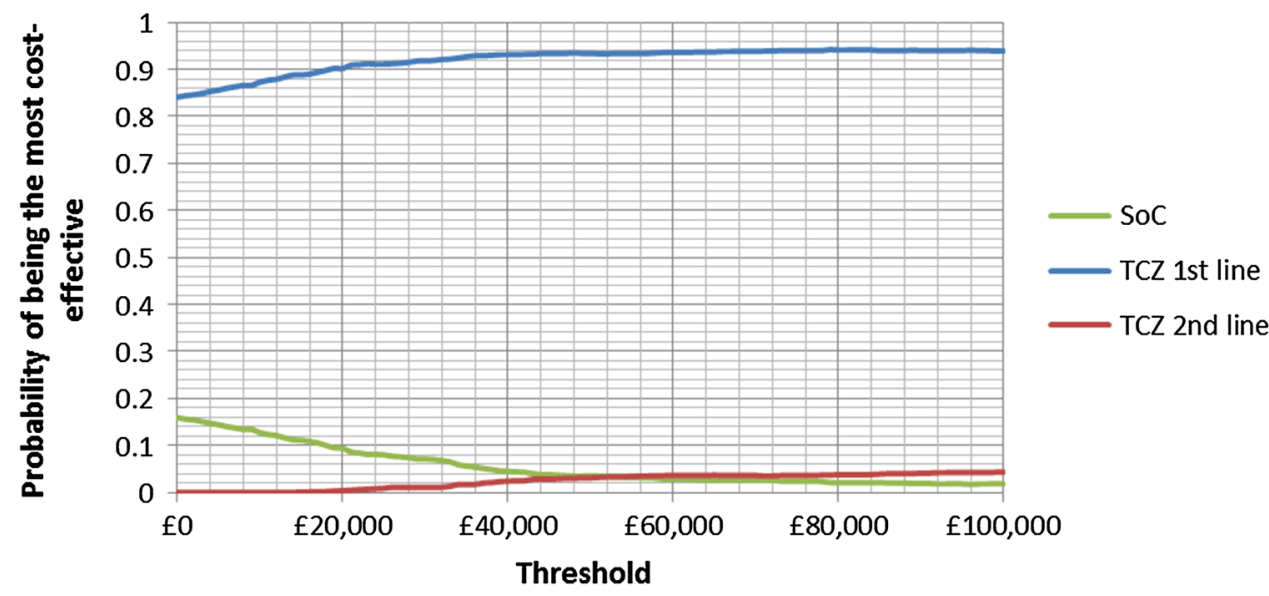

(patients who run out of treatment options), they tend to consider extreme examples in their responses. Furthermore, clinical practice is often determined by the number of treatments available to clinicians, which may vary a lot from one jurisdiction to another. Since the data survey on palliative care involved only six clinical experts and responses varied significantly, the results should be interpreted with caution.

Further research is necessary for a number of parameters of the economic model. In particular, with regard to discontinuation, one could explore different hypotheses that test treatment-related discontinuation, age, gender and other patient characteristics. Our analysis assumed that discontinuation was response related, which fitted well with our model structure and produced plausible results. Furthermore, as new and more bDMARDs become available to RA patients, research could identify subgroups where treatments are more efficacious, potentially producing more cost savings for the healthcare provider.

Overall, although we found that the model was sensitive to changes that affect the cost of TCZ and time on treatment, we believe that further refinement of those parameters would not significantly change the analysis conclusions.

\section{Conclusion}

The addition of TCZ to the SoC is estimated to be a costeffective strategy in the treatment of patients with moderate to severe RA. In 1,000 samples in the PSA, the strategy with TCZ at first line had a higher estimated probability of being cost effective than TCZ at second line.

Funding sources This study was funded by F. Hoffmann-La Roche Ltd. Roche was specifically involved in the analysis of clinical trial data. All other evidence was synthesised by independent analysts.

Conflict of interest disclosures $\mathrm{AF}$ and $\mathrm{TH}$ have disclosed that they have received funds as consultants of F. Hoffmann-La Roche
Ltd. AD, DS and LS are employees of a company that has received funds from the same. DN has disclosed that he has no conflicts of interest to declare. FD has disclosed that he is an employee of F. Hoffmann-La Roche Ltd.

Author contributions $\mathrm{AD}$ was responsible for the design and development of the model and led the overall economic analysis. AF led the analysis for treatment discontinuation, provided expert opinion on missing references and contributed to the palliative care survey and the TCZ clinical trial data input. TH provided expert opinion on missing references and contributed to the palliative care survey and the TCZ clinical trial data input. DS assisted in the synthesis of model inputs and the development of the model and manuscript. LS led the palliative care analysis and assisted in the writing of the manuscript. DN provided support in the treatment discontinuation analysis. FD assisted with the TCZ clinical trial data input and overall review of the manuscript. $\mathrm{AD}$ is the guarantor for the overall content of this manuscript.

Open Access This article is distributed under the terms of the Creative Commons Attribution Noncommercial License which permits any noncommercial use, distribution, and reproduction in any medium, provided the original author(s) and the source are credited.

\section{References}

1. Symmons D, Turner G, Webb R, et al. The prevalence of rheumatoid arthritis in the United Kingdom: new estimates for a new century. Rheumatology. 2002;41(7):793-800.

2. Doran MF, Crowson CS, Pond GR, et al. Predictors of infection in rheumatoid arthritis. Arthritis Rheum. 2002;46(9):2294-300.

3. Gabriel SE. Why do people with rheumatoid arthritis still die prematurely? Ann Rheum Dis. 2008;67(Suppl 3):iii30-4.

4. Menue C, Touze E, Ludovic Trinquart L, Allanore Y. Trends in cardiovascular mortality in patients with rheumatoid arthritis over 50 years: a systematic review and meta-analysis of cohort studies. Rheumatology. 2009;48(10):1309-13.

5. National Audit Offices. Services for people with rheumatoid arthritis. The Stationary Office. http://www.nao.org.uk/ publications/0809/services_for_people_with_rheum.aspx. Accessed Nov 2009.

6. Brouwer WB, van Exel NJ, Van de Berg B, et al. Burden of caregiving: evidence of objective burden, subjective burden, and 
quality of life impacts on informal caregivers of patients with rheumatoid arthritis. Arthritis Rheum. 2004;51(4):570-7.

7. Hallert E, Husberg M, Jonsson D, et al. Rheumatoid arthritis is already expensive during the first year of the disease (the Swedish TIRA project). Rheumatology (Oxford). 2004;43(11):1374-82.

8. Michaud K, Messer J, Choi HK, Wolfe F. Direct medical costs and their predictors in patients with rheumatoid arthritis: a threeyear study of 7,257 patients. Arthritis Rheum. 2003;48:2750-62.

9. NICE Assessment report. Adalimumab, etanercept, infliximab, certolizumab pegol, golimumab, tocilizumab and abatacept for the treatment of rheumatoid arthritis not previously treated with disease-modifying anti-rheumatic drugs and after the failure of conventional disease-modifying anti-rheumatic drugs only: systematic review and economic evaluation. Produced by ScHARR, University of Sheffield. August 2013.

10. NICE TA247 Tocilizumab for the treatment of rheumatoid arthritis (rapid review of technology appraisal guidance 198). February 2012.

11. Scottish Medicines Consortium. Re-submission tocilizumab, $20 \mathrm{mg} / \mathrm{mL}$, concentrate for solution for infusion (RoActemra ${ }^{\circledR}$ ) SMC No. (774/12). August 2012.

12. Gabriel S, Drummond M, Maetzel A, et al. OMERACT 6 Economics working group report: a proposal for a reference case for economic evaluation in rheumatoid arthritis. J Rheumatol. 2003;30:886-90.

13. NICE TA195 Adalimumab, etanercept, infliximab, rituximab and abatacept for the treatment of rheumatoid arthritis after the failure of a TNF inhibitor. August 2010.

14. Hyrich KL, Deighton C, Watson KD, et al. Benefit of anti-TNF therapy in rheumatoid arthritis patients with moderate disease activity. Rheumatology (Oxford). 2009;48(10):1323-7.

15. NICE TA130 Adalimumab, etanercept and infliximab for the treatment of rheumatoid arthritis. October 2007.

16. Wolfe F. The mortality of rheumatoid arthritis. Arthritis Rheum. 1994;37(4):481-94.

17. Buckley F. Presentation 2171-ACR 2012, 10-14 Nov, Washington D.C., USA. 2012.

18. Cohen SB, Emery P, Greenwald MW, et al. Rituximab for rheumatoid arthritis refractory to anti-tumor necrosis factor therapy. Arthritis Rheum. 2006;54(9):2793-806.

19. Fleischmann RM, Halland AM, Brzosko M, et al. Tocilizumab inhibits structural joint damage and improves physical function in patients with rheumatoid arthritis and inadequate responses to methotrexate: LITHE study 2-year results. J Rheumatol. 2013;40(2):113-26.

20. Genovese MC, McKay JD, Nasonov EL, et al. Interleukin-6 receptor inhibition with tocilizumab reduces disease activity in rheumatoid arthritis with inadequate response to disease-modifying antirheumatic drugs: the tocilizumab in combination with traditional disease-modifying antirheumatic drug therapy study. Arthritis Rheum. 2008;58(10):2968-80.

21. Smolen JS, Beaulieu A, Rubbert-Roth A, et al. Effect of interleukin-6 receptor inhibition with tocilizumab in patients with rheumatoid arthritis (OPTION study): a double-blind, placebocontrolled, randomised trial. Lancet. 2008;371(9617):987-97.

22. Stevenson M, Archer R, Tosh J, et al. Adalimumab, etanercept, infliximab, certolizumab pegol, golimumab, tocilizumab and abatacept for the treatment of rheumatoid arthritis not previously treated with disease-modifying anti-rheumatic drugs and after the failure of conventional disease-modifying anti-rheumatic drugs only: systematic review and economic evaluation. National Institute of Clinical Excellence. August 2013.

23. Gulfe A, Geborek P, Saxne T. Response criteria for rheumatoid arthritis in clinical practice: how useful are they? Ann Rheum Dis. 2005;64(8):1186-9.
24. Soliman MM, Ashcroft DM, Watson KD, et al. Impact of concomitant use of DMARDs on persistence with anti-TNF therapies in patients with rheumatoid arthritis: results from the British Society for Rheumatology Biologics Register. Ann Rheum Dis. 2011;70(4):583-9.

25. Brennan A, Bansback N, Reynolds A, Conway P. Modelling the cost-effectiveness of etanercept in adults with rheumatoid arthritis in the UK. Rheumatology (Oxford). 2004;43(1):62-72.

26. Singh JA, Wells GA, Christensen R, et al. Adverse effects of biologics: a network meta-analysis and Cochrane overview. Cochrane Database Syst Rev. 2011;(2):CD008794.

27. Furst DE, Schiff MH, Fleischmann RM, et al. Adalimumab, a fully human anti tumor necrosis factor-alpha monoclonal antibody, and concomitant standard antirheumatic therapy for the treatment of rheumatoid arthritis: results of STAR (Safety Trial of Adalimumab in Rheumatoid Arthritis). $\mathrm{J}$ Rheumatol. 2003;30(12):2563-71.

28. Kremer JM, Genant HK, Moreland LW, et al. Effects of abatacept in patients with methotrexate-resistant active rheumatoid arthritis: a randomized trial. Ann Intern Med. 2006;144(12): 865-76.

29. Sandborn WJ, Feagan BG, Stoinov S, et al. Certolizumab pegol for the treatment of Crohn's disease. N Engl J Med. 2007;357: 228-38.

30. Schiff MH, DiVittorio G, Tesser J, et al. The safety of anakinra in high-risk patients with active rheumatoid arthritis: six month observations of patients with comorbid conditions. Arthritis Rheum. 2004;50(6):1752-60.

31. Westhovens R, Yocum D, Han J, et al. The safety of infliximab, combined with background treatments, among patients with rheumatoid arthritis and various comorbidities: a large, randomized, placebo-controlled trial. Arthritis Rheum. 2006;54(4):1075-86.

32. Alava MH, Wailoo AJ, Ara R. Tails from the peak district: adjusted limited dependent variable mixture models of EQ-5D questionnaire health state utility values. Value Health. 2012;15(3):550-61.

33. Sisk J, Maskowitz AJ, Whang W, et al. Cost-effectiveness of vaccination against pneumococcal bacteremia among elderly people. JAMA. 1997;278(16):1333-9.

34. Ara R, Brazier J. Populating an economic model with health state utility values: moving towards better practice. Value Health. 2010;13(5):509-18.

35. British National Formulary. British National Formulary 63. Retrieved 1 June 2012, from Medicines Complete. 2012. http:// www.medicinescomplete.com.

36. Electronic Medicines Compendium (eMC). SPC. Retrieved 1 June 2012, from Electronic Medicines Compendium. 2012. http:// www.medicines.org.uk.

37. Barton P, Jobanputra P, Wilson J, et al. The use of modelling to evaluate new drugs for patients with a chronic condition: the case of antibodies against tumour necrosis factor in rheumatoid arthritis. Health Technol Assess. 2004;8(11):iii, 1-91.

38. NHS reference costs 2010/2011. Department of Health. Publications policy and guidance. Retrieved 1 March 2012, from Department of Health. 2011. http://www.dh.gov.uk.

39. Kobelt G, Eberhardt K, Jönsson L, Jönsson B. Economic consequences of the progression of rheumatoid arthritis in Sweden. Arthritis Rheum. 1999;42(2):347-56.

40. Kobelt G, Lindgren P, Lindroth Y, et al. Modelling the effect of function and disease activity on costs and quality of life in rheumatoid arthritis. Rheumatology (Oxford). 2005;44(9): 1169-75.

41. NICE. Guide to the methods of technology appraisal. April 2013.

42. Boggs R, Sengupta N, Ashraf T. Estimating health utility from a physical function assessment in rheumatoid arthritis (RA) 
patients treated with adalimumab (HUMIRA). Value Health. 2002;5(6):452.

43. Bansback N, Brennan A, Ghatnekar O. Cost effectiveness of adalimumab in the treatment of patients with moderate to severe rheumatoid arthritis in Sweden. Ann Rheum Dis. 2005;64(7): 995-1002.
44. Ducournau P, Kielhorn A, Wintfeld N. Comparison of linear and non-linear utility mapping between HAQ and EQ-5D using pooled data from the tocilizumab trials OPTION and LITHE. Annual Meeting of the British Society of Rheumatology (BSR), Glasgow. 2009; poster 258. 